\title{
When chaos meets hyperchaos: 4D Rössler model
}

\author{
Roberto Barrio ${ }^{\mathrm{a}, \mathrm{b}, *}$, M. Angeles Martínez ${ }^{\mathrm{b}}$, Sergio Serrano ${ }^{\mathrm{a}, \mathrm{b}}$, Daniel Wilczak ${ }^{\mathrm{c}}$ \\ ${ }^{a}$ Departamento de Matemática Aplicada and IUMA. University of Zaragoza, E-50009 Zaragoza, Spain. \\ ${ }^{b}$ Computational Dynamics group. University of Zaragoza, E-50009 Zaragoza, Spain. \\ ${ }^{c}$ Faculty of Mathematics and Computer Science. Jagiellonian University. Eojasiewicza 6, 30-348 Kraków, Poland.
}

\begin{abstract}
Chaotic behavior is a common feature of nonlinear dynamics, as well as hyperchaos in high dimensional systems. In numerical simulations of these systems it is quite difficult to distinguish one from another behavior in some situations, as the results are frequently quite "noisy". We show that in such systems a global hyperchaotic invariant set is present giving rise to long hyperchaotic transient behaviors. This fact provides a mechanism for these noisy results. The coexistence of chaos and hyperchaos is proved via Computer Assisted Proofs techniques.
\end{abstract}

Keywords: chaos, hyperchaos, Computer Assisted Proofs, hyperchaotic saddle PACS: 05.45.Ac, 05.45.Pq

\section{Introduction}

It's only a few decades since famous discovery of Lorenz [1] that deterministic systems can exhibit sensitive dependencies on initial conditions. However, a large number of researchers have been working deeply in the development of theoretical basements needed for the analysis of chaotic systems. A recent cornerstone theoretical result was the Tucker's computer-assisted proof of the existence (and of the mathematical structure) of the Lorenz chaotic attractor [2-4]. Furthermore, it has been shown that these systems reproduce nicely complex behaviors found in real systems of diverse nature [5-11]. Most of the results have been stated in three dimensional models, where, due to the restricted phase-space only low-dimensional chaos can be observed. The remaining main question is: What changes when higher dimensional systems are analyzed?

Chaotic systems are characterized by (at least) one direction of exponential spreading. A common way to detect this circumstance is by calculating the maximum Lyapunov exponent [12] of the orbit. If it is positive, the orbit exhibits sensitive dependence on initial conditions, and this is a standard indication of chaotic behaviour (we remark that a positive Lyapunov exponent is not always an indication of chaos, as shown, for instance, in [13-16]). If the number of directions of spreading is greater than one, the behavior of the system is hyperchaotic [17]. To detect this situation it becomes necessary to calculate more Lyapunov exponents and to determine how many of them are positive. Note that for continuous autonomous dynami-

\footnotetext{
* Corresponding author.

Email addresses: rbarrio@unizar.es (Roberto Barrio), gelimc@unizar.es (M. Angeles Martínez), sserrano@unizar.es (Sergio Serrano), wilczak@ii.uj.edu.pl (Daniel Wilczak)

cal systems, chaos can appear in systems with dimension greater than or equal to three.

The behavior (and therefore its analysis) of hyperchaotic systems is much more complicated than the case of systems with just a single positive Lyapunov exponent. There are two main reasons, firstly the need for a fourth dimension to the appearance of hyperchaos, which makes some tools of analysis for three-dimensional chaotic models not valid; on the other hand, the existence of more than one direction of spreading allows the system undergoes a broader spectrum of bifurcations. However, in practical applications it is necessary to model problems with dimension higher than three, in some of which hyperchaotic behavior appears like in mathematical models of electroencephalograms, chemical systems, electronic circuits [18-21] and in most of the networks of basic continuous systems, as coupled Lorenz or Rössler systems [22]. Besides, note that contemporary numerical weather prediction schemes are based on ensemble forecasting. Ensemble members are obtained by taking different (perturbed) models started with different initial conditions, an example of such kind of systems is the Lorenz-96 model [23]. In all these kinds of systems the appearance of hyperchaos is quite natural due to their dimension. In addition, this high dependence on initial conditions experienced by hyperchaotic systems has practical applications, such as encryption of information $[24,25]$.

That is why in the last two decades many articles have appeared in which the authors study dynamical systems with hyperchaotic behavior [26-30]. Many of them focus on the transition from chaotic to hyperchaotic behavior. The problem is that the numerical study of these systems is some times no clear at all, giving really a confuse analysis about if the system is chaotic or hyperchaotic [31]. 
Therefore, one of the main goals of this paper is to study in detail the main reason of why most of these studies really fail in giving a clear picture of what happens in the system. We show that the main behavior of these systems is hyperchaotic, but it can be a transient behavior or an attracting one. This duality is rigorously established via Computer Assisted Proofs (CAP) techniques and it gives a mechanism for the "noisy" simulations in many studies [31].

The paper is organized as follows. In Section 2 we present several numerical simulations on the 4D Rössler model to study the appearance of chaotic and hyperchaotic behaviours and how the results depend on the way of computing the Lyapunov exponents. In Section 3 we give the basic steps of a Computer-Assisted proof of the coexistence of chaotic and hyperchaotic behaviour, giving in some situations the existence of long hyperchaotic transients that may give rise to "noisy" numerical simulations. Finally, in Section 4 we present some conclusions.

\section{Chaos and hyperchaos: numerical studies.}

Our first question is to study the detection of the different behaviours (regular, chaotic or hyperchaotic) of the dynamical models. The usual approach is to calculate two or more dominant Lyapunov exponents and determine how many of them are positive. Along this paper, we will use, as paradigmatic example, the well-known 4D Rössler model [17], given by:

$$
\left\{\begin{array}{l}
\dot{x}=-(y+z) \\
\dot{y}=x+a y+w \\
\dot{z}=b+x z \\
\dot{w}=-c z+d w
\end{array}\right.
$$

where we fix the values of parameters $b=3.0$ and $d=0.05$, and we allow to change the values of $a$ and $c$. This model was the first model where it was shown the existence of hyperchaotic behaviour.

In Fig. 1 we present two biparametric plots showing the different behaviours based on the Lyapunov exponents computed using the algorithm of Wolf et al. [32]. The only difference in the simulations is that the lower picture is done considering a transient time $3 \times 10^{4}$ before computing the exponents. In the simulations we have differentiated the cases of having two large positive Lyapunov exponents (strong hyperchaos) with the case of having two positive values but one of them quite small (weak hyperchaos). The colors in the figure determine the different behaviors detected in the simulations. White represents a limit cycle, maximum Lyapunov exponent $\lambda_{1}=0$ and the others $\lambda_{2,3,4}<0$; blue for torus, $\lambda_{1,2}=0$ and $\lambda_{3,4}<0$; red for chaotic, $\lambda_{1}>0, \lambda_{2}=0$ and $\lambda_{3,4}<0$; green for weak hyperchaos (see Fig. 3), $0.05>\lambda_{1}>\lambda_{2}>0, \lambda_{3}=0$, $\lambda_{4}<0$; brown for strong hyperchaos, $\lambda_{1}>0.05>\lambda_{2}>0$, $\lambda_{3}=0, \lambda_{4}<0$. Comparing both pictures, we can see how the upper picture is completely dominated by brown
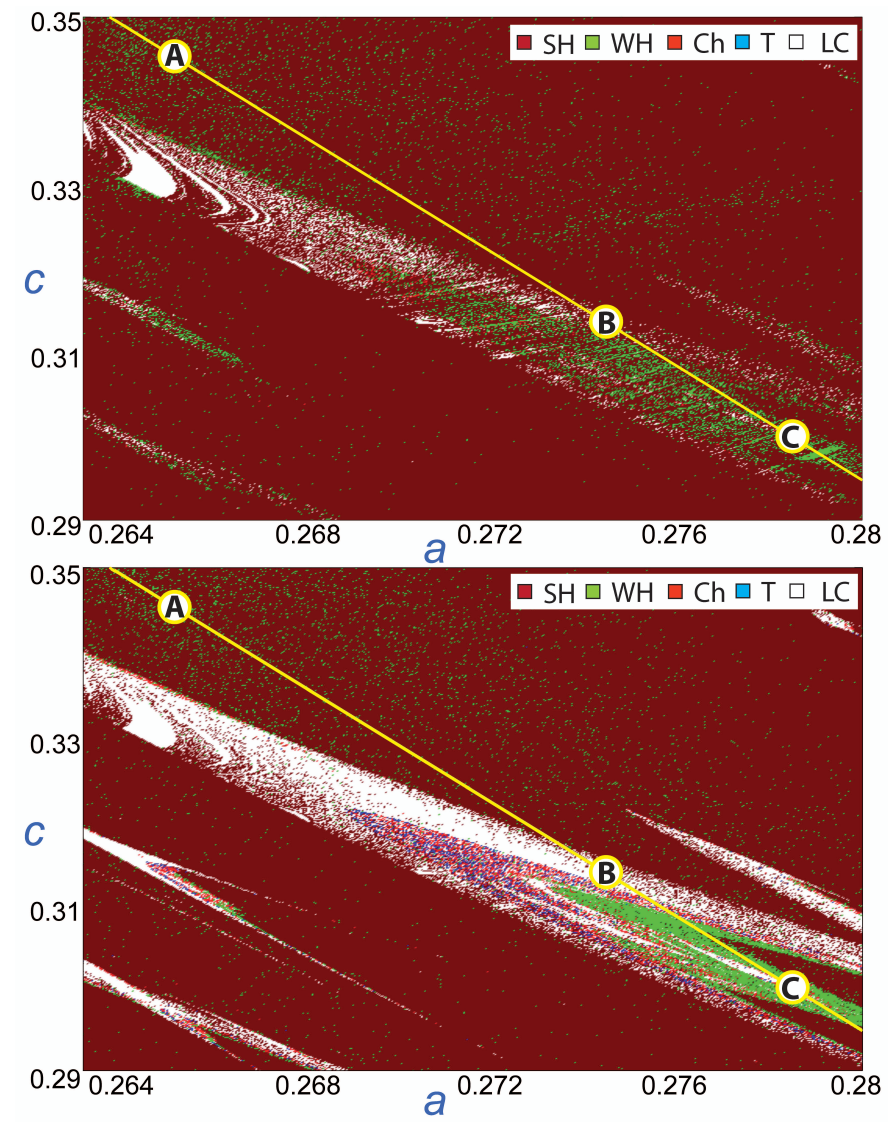

Figure 1: Lyapunov exponents biparametric plots showing periodic (limit cycles, LC), quasiperiodic (torus, T), chaotic (Ch), weakhyperchaotic (WH) and strong-hyperchaotic ( $\mathrm{SH}$ ) behaviors. (Top) without transient time in the simulations and (bottom) with transient time.

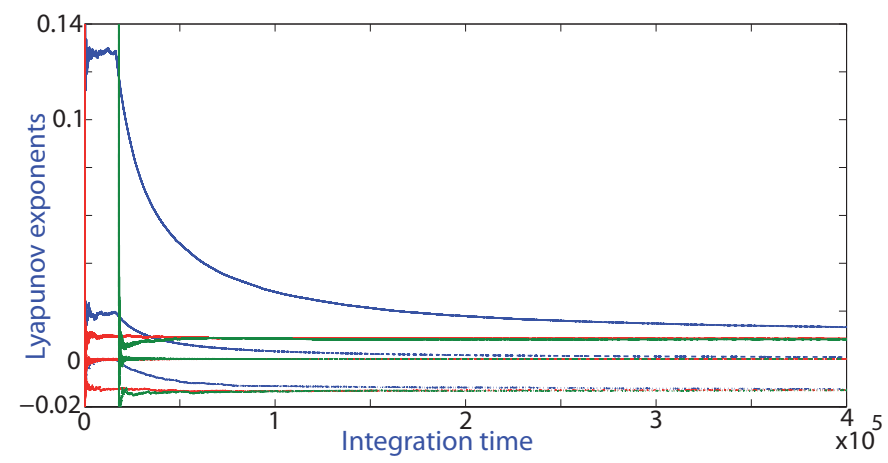

Figure 2: Time evolution of the Lyapunov exponents depending on using or not transient time and depending on the initial conditions. Red, without transient time but with initial conditions that go directly to the chaotic attractor; blue, without transient time, with initial conditions that go first close to the hyperchaotic saddle; green, with transient time.

color, representing hyperchaotic behavior. Note that in almost all the results we have a "noisy" picture without giving a clear study of the real behavior, especially in the upper plot. This situation appears also in most of the simulations in literature [31]. In contrast, in the lower picture, wherein we have used the transient time, those 


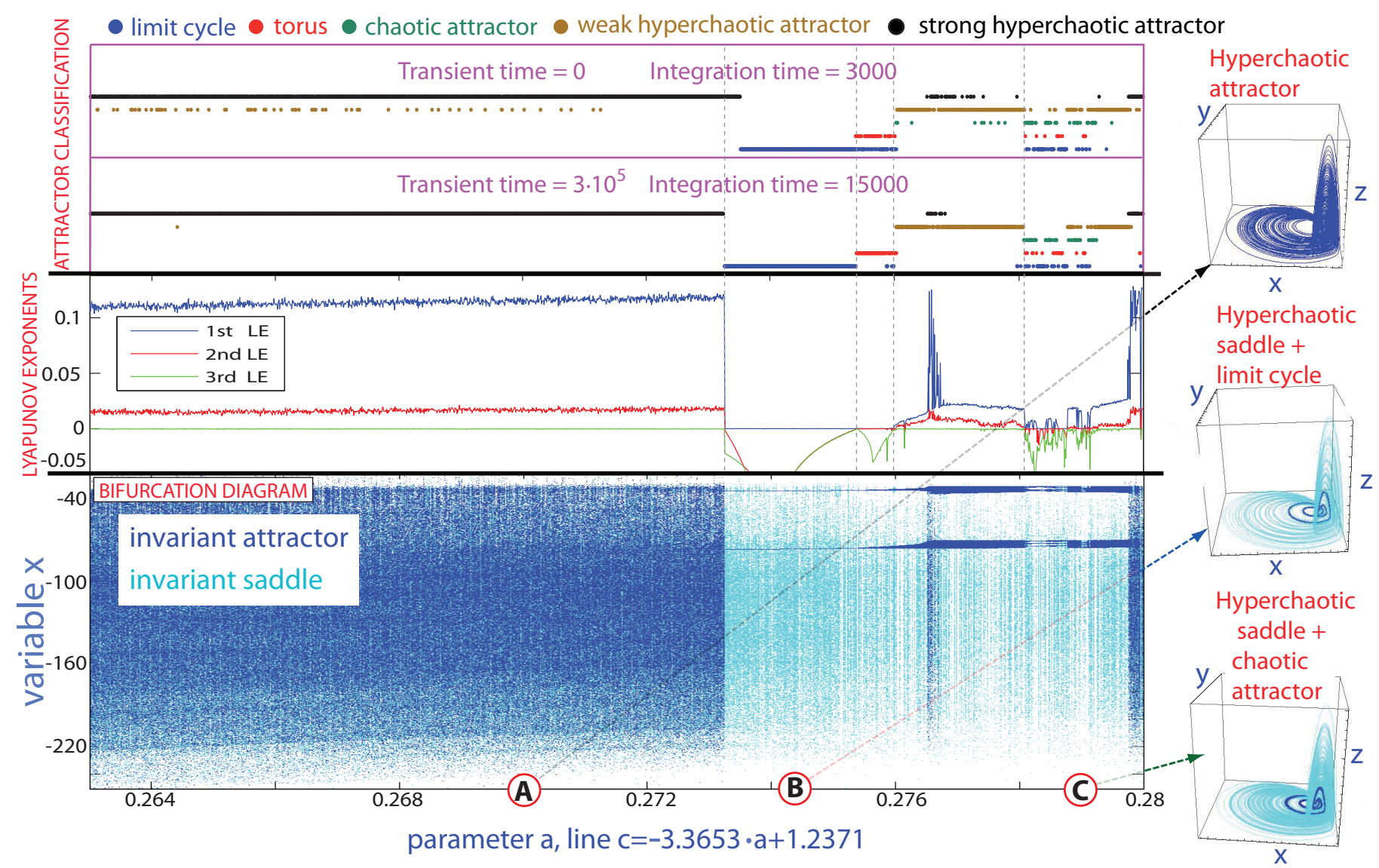

Figure 3: Top: classification of the attractors depending on the integration and transient time. Middle: Lyapunov exponents in the selected line. Bottom: bifurcation diagram along the same line (changing parameter $a$ ). Dark blue corresponds to the invariant attractor and light blue to the invariant saddle. On the right, three examples of the hyperchaotic saddle together with the corresponding attractor.

structures that were hardly visualized in the upper picture, now appear in a clearer way, but still some "noisy" patterns appear. The integration time (without considering the transient time) used in both pictures is 3000, the first question is whether the upper picture, at least, correctly identifies the behavior of the system at that final moment. Fig. 2 shows how the calculation of exponents keeps memory of past behavior, taking time to recognize behavior changes [33]. This tells us that if we want to classify the behavior of the system at any given time, we must try to start the calculation of Lyapunov exponents from a time at which the system experiences such behavior. If we want to study the type of attractor which goes into the dynamics of the system, then we have to consider a sufficiently long transient time. We can see that the second picture of Fig. 1 still shows "noise", so that higher transient and/or integration time is necessary to get an absolutely clear picture. In a more detailed study [34], it has been found that a transient time equal to $3 \times 10^{5}$ and an integration time equal to $1.5 \times 10^{4}$ are values needed to obtain figures with a significant "noise" reduction. Higher values provide little improvement that is not worth the cost in computational time involved. However, these values would imply that the corresponding picture in Fig. 1 (with a mesh of 500 by 500) would cost 189 days on an Intel Core2 Duo E6750 @ 2.66GHz (compared to 4.1 days for the upper picture and 21 days for the picture below). This observation permits to perform better simulations of Lyapunov exponents, but in any case it does not provide a complete explanation of the "noisy" patterns and why we need such a long transient time in this system.

In order to study in detail what really happens, we focus our attention to a line, $c(a)=-3.3653 \cdot a+1.2371$, in the biparametric picture (Fig. 1), because it crosses regions with all the different behaviors detected in the model. In Fig. 3 we show on the top the classification according to the type of behavior encountered through calculation of Lyapunov exponents using two different integration and transient times. We show that for low integration times more hyperchaotic situations are detected. A detailed analysis is shown in the middle plot. Note that we observe some of the familiar routes to hyperchaos, like tangencies among the hyperchaotic saddle and a periodic orbit (fold bifurcations of limit cycles) or smooth growing of the second Lyapunov exponent, as pointed in [35, 30]. On the bottom plot we show the bifurcation diagram along the same line and on the right plots the attractors and the hyperchaotic saddle at three points (the same pointed out 
in Fig. 1). The most interesting fact is the global existence of a hyperchaotic invariant set along all the line (and in fact along all the biparametric simulations of Fig. 1). This figure establishes a clear reason for the "noisy" simulations, all along the parametric phase space there is a global hyperchaotic invariant, sometimes a saddle structure and sometimes a global attractor, but in any case it gives a long transient hyperchaotic behavior.

\section{Chaos and hyperchaos: Computer-Assisted proof results.}

The above numerical study has allowed us to generate hypothesis about the existence of a hyperchaotic saddle, coexistence of chaotic and hyperchaotic behavior and different kinds of attractors. Moreover, the global existence of the hyperchaotic invariant set gives an explanation of the difficulty in most of the numerical simulations in literature to provide with a clear study of the behavior of the systems. As to obtain an analytical proof of this fact is not possible (or at least quite complex), we give some computer-assisted proofs, thanks to the use of the CAPD library [36] of rigorous computing and the techniques developed in [37-40]. To that goal, we take the parameter values $a=0.27857, b=3, c=0.3$ and $d=0.05$, where we have numerically detected the coexistence of a hyperchaotic saddle and a chaotic attractor, and we define a Poincaré section $\Pi=\left\{(x, 0, z, w) \in \mathbb{R}^{4} ; \dot{y}=x+w<0\right\}$ and the associated Poincaré map $P: \Pi \rightarrow \Pi$. Then we run the algorithm ENCLOSEForwardTrajeCtory (see [40, Algorithm 1]) on a computer with 64 CPUs. The program stopped after 162 minutes (which gives circa $173 \mathrm{CPU}$ hours) using at peek $30 \mathrm{~GB}$ of memory and returned a positive invariant set $B$ for $P$ which is the union of 22685758 $3 \mathrm{D}$ parallelograms of size $\left(2^{-10}, 2^{-10}, 2^{-12}\right)$. This proves (see [40, Lemma 3.5]) that $B$ is a trapping region for $P$, namely $P(B) \subset B$. Projection of $B$ onto $(x, z)$ plane is shown in Fig. 4.

There are many efficient algorithms for finding short cycles for maps - see for instance [41, 42]. As finding all short cycles is not the primary goal of this paper we used a much easier strategy. The center of each box forming the trapping region $B$ has been used as a seed point for the standard Newton-based multiple shooting method. This strategy returned a quite large amount of approximate periodic points, with one or two unstable directions, among which the shortest were of period 8. From this set we selected six unstable periodic orbits (UPOs) named $\left\{c_{1}^{8}, c_{2}^{8}, c_{3}^{8}, c_{4}^{16}, h_{1}^{12}, h_{2}^{14}\right\} \subset B$, where the superscript stands for the period of the point. This particular choice has been done manually after many numerical simulations and for the reason explained in the sequel. Using the Interval Newton Operator and Interval Krawczyk Operator we were able to prove that in a ball of radius $10^{-8}$ centered at each approximate periodic point there exists unique periodic point of the same period. Then we computed rigorous bounds for the eigenvalues of the derivative

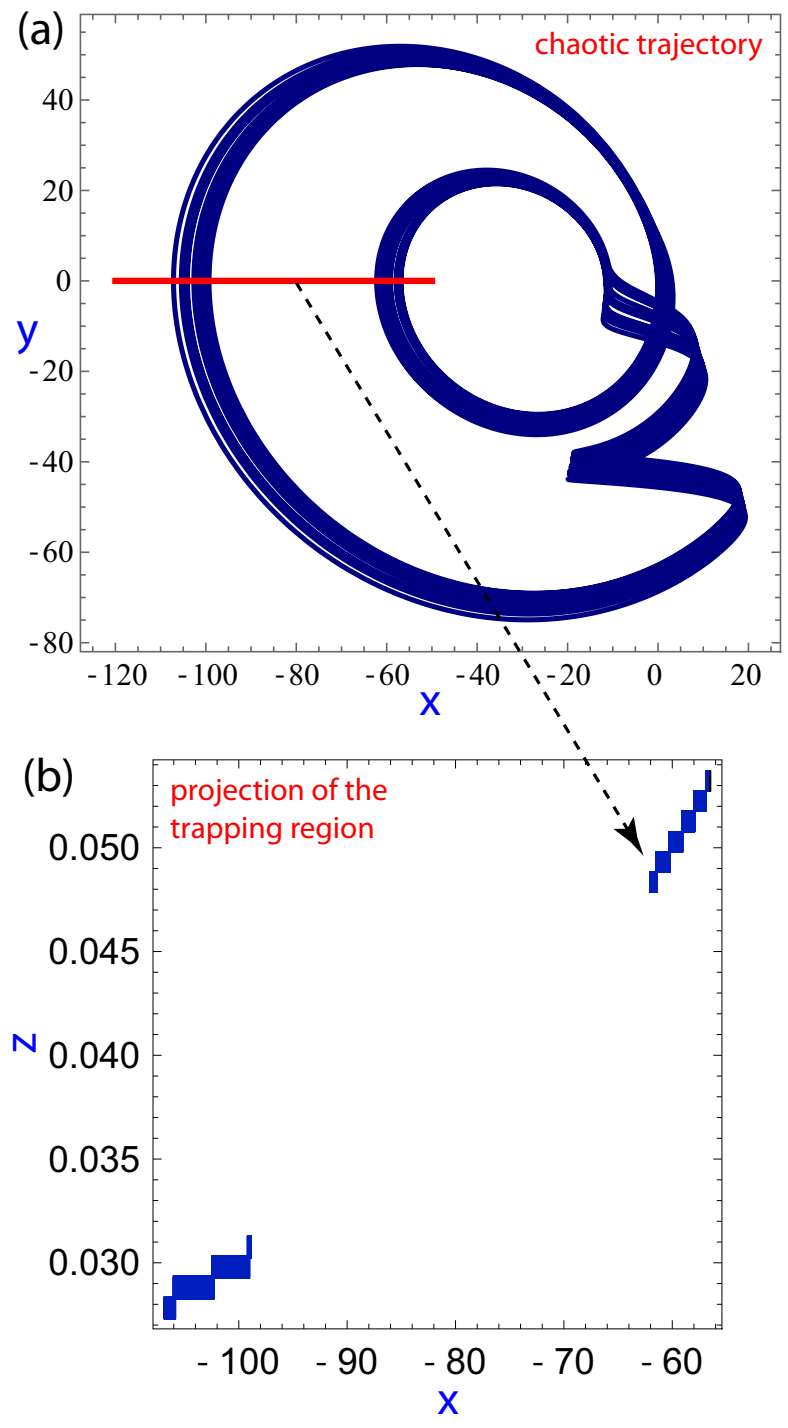

Figure 4: Top: chaotic trajectory for the hyperchaotic Rössler system and the Poincaré section (red line). Bottom: rigorous projection of the trapping region for the Poincaré map.

of $P^{j}$ at each periodic point which allowed us to determine its stability type. The orbits $c_{i}^{j}$ have one-dimensional unstable manifold and those corresponding to $h_{i}^{j}$ have twodimensional unstable manifold.

On each pair of UPOs $\left(c_{1}^{8}, c_{2}^{8}\right),\left(c_{3}^{8}, c_{4}^{16}\right)\left(h_{1}^{12}, h_{2}^{14}\right)$ we constructed a horseshoe dynamics using the method of covering relations $[43,44]$ in a similar way as in [39] and we proved its existence by means of the algorithms COMPUteUnstableWALl and COMPUTEBoundary - see [38, Algorithms 1,2]. Then, applying a cone condition criterion introduced in [37] we verified the existence of countable infinity of heteroclinic and homoclinic orbits linking some of the above periodic orbits. All the computations related to the existence of chaotic, hyperchaotic and linking dynamics were performed on a computer with 64 CPUs and they have been completed within 237 minutes. In Fig. 5 we show some approximate trajectories of the hetero- 

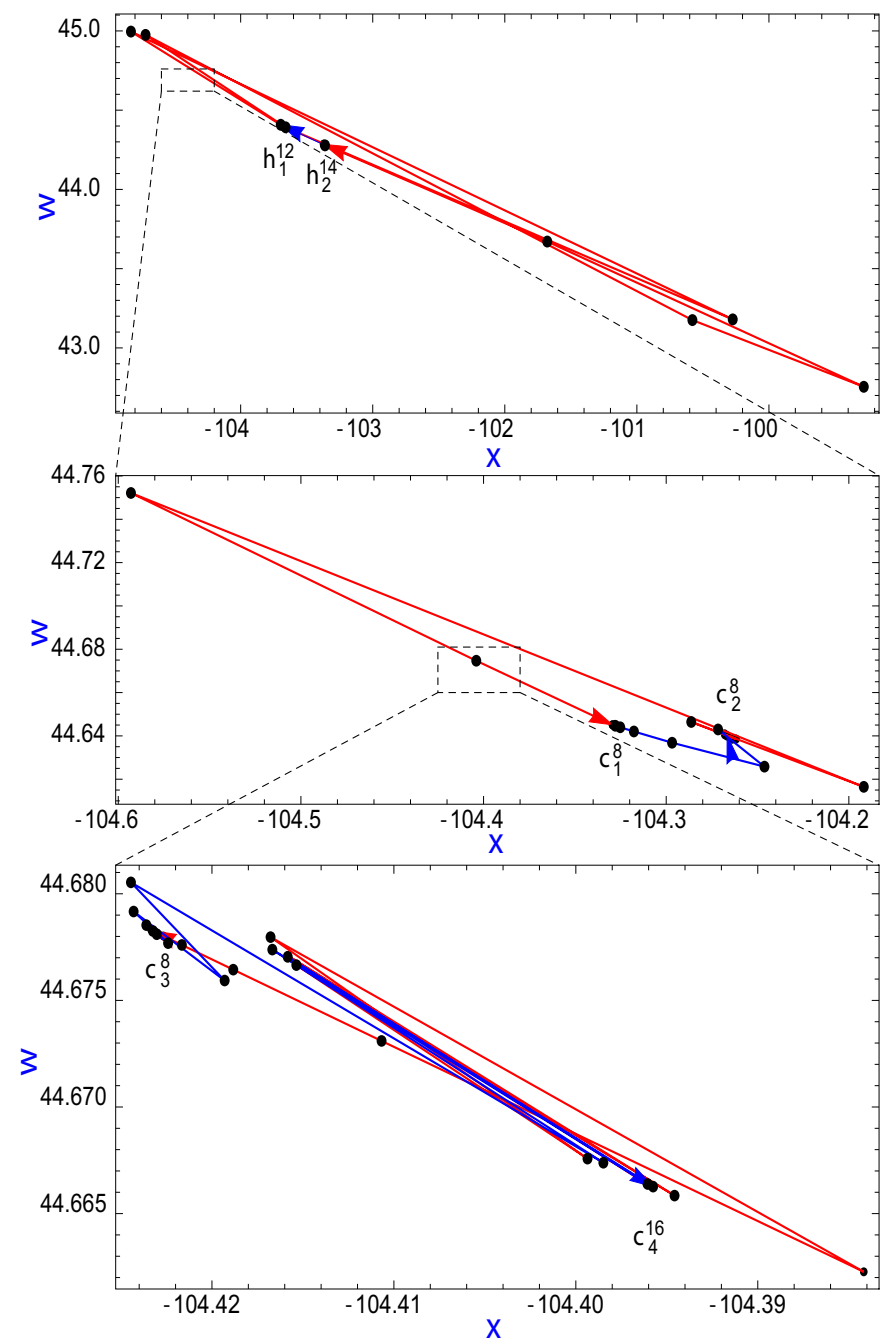

Figure 5: Approximate trajectories of the heteroclinic connections among the points of the UPOs $\left\{c_{1}^{8}, c_{2}^{8}, c_{3}^{8}, c_{4}^{16}, h_{1}^{12}, h_{2}^{14}\right\}$.

clinic connections obtained with the CAPD library. Note that the existence is rigorously proved via CAP techniques, although the trajectories are approximate (in a small interval). The location of homoclinic orbits is, by itself, a complicate task [45], but what we have done is going a step further giving a CAP proof of their existence.

The following list summarizes proved results, for the particular set of parameters chosen in this paper, and illustrated on Fig. 6 (complete details will appear in a more comprehensive study [34]):

- there is an explicitly given trapping region $B \subset \Pi$ for $P$, i.e. $P(B) \subset B$,

- the maximal invariant set $A=\operatorname{inv}(P, B)$ contains three invariant sets, say $S_{1}, S_{2}, S_{3}$, on which the dynamics is $\Sigma_{2}$ chaotic, i.e. it is semiconjugated to the Bernoulli shift on two symbols,

- $S_{1}$ is a hyperchaotic set with two positive Lyapunov exponents,

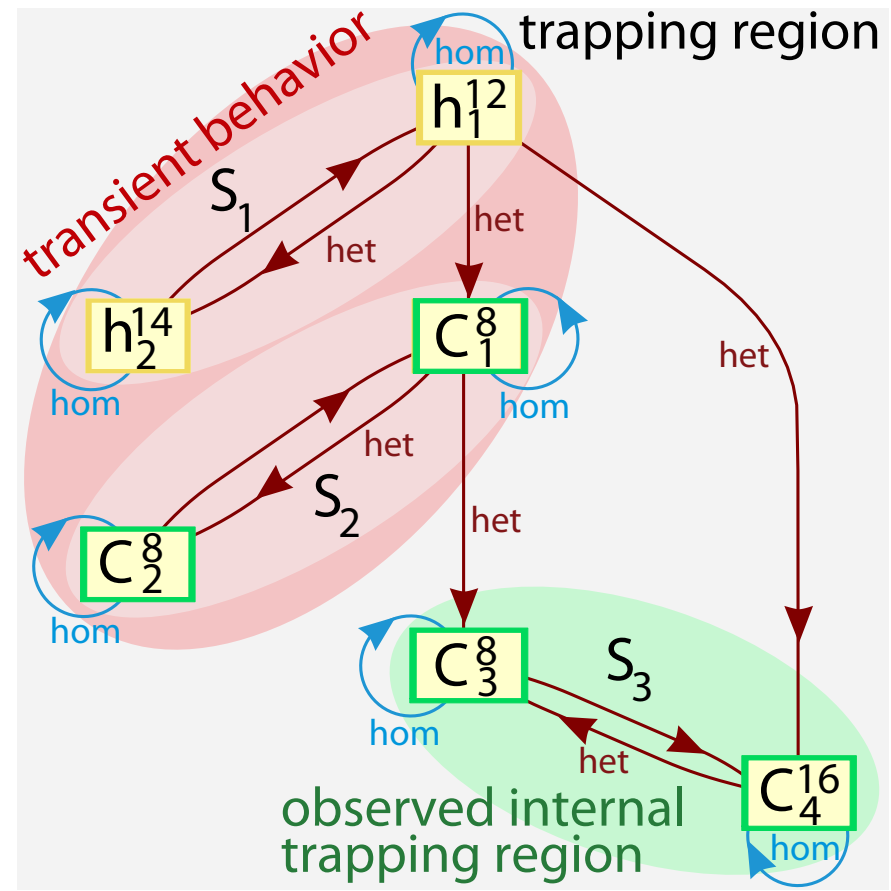

Figure 6: Scheme of the connections between the different invariant sets that control the symbolic dynamics for the hyperchaotic Rössler system. The green oval surrounding $S_{3}$ set is an observed internal trapping region that forbids backward connections from $S_{3}$ to $S_{2}$ and $S_{1}$. Although the presence of such internal trapping region is observed in numerical simulations (giving a numerically observed chaotic attractor) we could not prove its existence by means of computer assisted techniques.

- $S_{2}$ and $S_{3}$ are chaotic sets with one positive Lyapunov exponent,

- there is a countable infinity of periodic orbits of arbitrary large periods (every periodic path on the graph presented in Fig. 6 is realized by a periodic orbit)

- there is a countable infinity of heteroclinic/homoclinic orbits linking periodic orbits inside each horseshoe, and a countable infinity of heteroclinic connections linking $S_{1}$ with $S_{2}, S_{2}$ with $S_{3}$ and $S_{1}$ with $S_{3}$. In fact, every finite and nonconstant path on the graph presented in Fig. 6 is realized by a homoclinic/heteroclinic connection between periodic orbits at the beginning and at the end of this sequence.

Although the results are proved for just one set of parameters, we note that all the methods used in computer assisted proofs are robust under perturbations. Therefore the same statements hold true for all parameter values from some (unknown) neighborhood of the selected by the authors. We remark that in all the numerical simulations done by the authors we can observe the same picture, supporting these statements as a common situation in the system. Note also that we have proved the existence of hyperchaotic and chaotic invariant sets which give a partial description of the dynamics in the trapping region $B$. One may expect the existence of a chaotic attractor or 
even an attracting periodic point of (perhaps) very high period that is not distinguishable from chaotic solutions even in very accurate numerical simulation.

\section{Conclusions}

We have shown that in generic hyperchaotic systems one may expect to have a global hyperchaotic behavior, sometimes being a hyperchaotic attractor or a hyperchaotic saddle giving rise to possible long hyperchaotic transient behavior. This hyperchaotic invariant is constructed via UPOs with two-dimensional (or higher) unstable manifolds and their homo- and heteroclinic connections. The existence also of UPOs with just a one-dimensional unstable manifold creates a gradient like structure giving routes that connect hyperchaos $\rightarrow$ chaos $\rightarrow$ chaos $\rightarrow$ attractor (chaotic or not). Depending on how these invariants are connected we have one kind of attractor or another, but what we have is that chaotic and hyperchaotic behavior is always present. This structure justifies numerical simulations in literature that generate quite noisy results making difficult to differentiate chaotic from hyperchaotic behavior.

\section{Acknowledgements}

R.B., S.S. have been supported during this research by the Spanish Research Grant MTM2012-31883. D.W. has been supported by the Polish Ministry of Science and Higher Education, Grant no. 2011/03/B/ST1/04780.

[1] E. Lorenz, Deterministic nonperiodic flow, J. Atmospheric Sci. 20 (1963) 130-141.

[2] V. Afraimovic, V. V. Bykov, L. P. Shilnikov, The origin and structure of the Lorenz attractor, Sov. Phys. Dokl. 22 (1977) 253-255.

[3] J. Guckenheimer, R. F. Williams, Structural stability of Lorenz attractors, Inst. Hautes Études Sci. Publ. Math. 50 (1) (1979) $59-72$.

[4] W. Tucker, A rigorous ODE solver and Smale's 14th problem, Found. Comput. Math. 2 (1) (2002) 53-117.

[5] T. Shinbrot, W. Ditto, C. Grebogi, E. Ott, M. Spano, J. A. Yorke, Using the sensitive dependence of chaos (the "butterfly effect") to direct trajectories in an experimental chaotic system, Phys. Rev. Lett. 68 (19) (1992) 2863-2866.

[6] C. Bonatto, J. C. Garreau, J. A. C. Gallas, Self-Similarities in the Frequency-Amplitude Space of a Loss-Modulated $\mathrm{CO}_{2}$ Laser, Phys. Rev. Lett. 95 (2005) 143905.

[7] R. Barrio, F. Blesa, S. Serrano, Qualitative analysis of the Rössler equations: Bifurcations of limit cycles and chaotic attractors, Phys. D 238 (2009) 1087-1100.

[8] R. Stoop, P. Benner, Y. Uwate, Real-World Existence and Origins of the Spiral Organization of Shrimp-Shaped Domains, Phys. Rev. Lett. 105 (2010) 074102.

[9] S. Serrano, R. Barrio, A. Dena, M. Rodríguez, Crisis curves in nonlinear business cycles, Communications in Nonlinear Science and Numerical Simulation 17 (2) (2012) 788-794.

[10] R. Barrio, F. Blesa, S. Serrano, Topological Changes in Periodicity Hubs of Dissipative Systems, Phys. Rev. Lett. 108 (2012) 214102.

[11] R. Barrio, M. A. Martinez, S. Serrano, A. Shilnikov, Macroand micro-chaotic structures in the Hindmarsh-Rose model of bursting neurons, Chaos 24 (2) 023128.
[12] K. T. Alligood, T. Sauer, J. A. Yorke, Chaos: an introduction to dynamical systems, Textbooks in mathematical sciences, Springer, 1996.

[13] P. Cvitanovic, R. Artuso, R. Mainieri, G. Tanner, G. Vattay, Chaos: Classical and quantum, ChaosBook. org (Niels Bohr Institute, Copenhagen 2005) .

[14] G. A. Leonov, N. V. Kuznetsov, Time-Varying Linearization and the Perron effects, International Journal of Bifurcation and Chaos 17 (04) (2007) 1079-1107.

[15] N. Kuznetsov, T. Mokaev, P. Vasilyev, Numerical justification of Leonov conjecture on Lyapunov dimension of Rossler attractor, Communications in Nonlinear Science and Numerical Simulation 19 (4) (2014) 1027-1034.

[16] N. V. Kuznetsov, T. A. Alexeeva, G. A. Leonov, Invariance of Lyapunov characteristic exponents, Lyapunov exponents, and Lyapunov dimension for regular and non-regular linearizations, http://arxiv.org/pdf/1410.2016.pdf .

[17] O. Rossler, An equation for hyperchaos, Physics Letters A $71(2-3)(1979) 155-157$.

[18] T. Matsumoto, L. O. Chua, K. Kobayashi, Hyperchaos: laboratory experiment and numerical confirmation, IEEE Trans. Circuits and Systems 33 (11) (1986) 1143-1149.

[19] R. Stoop, J. Peinke, J. Parisi, B. Röhricht, R. Huebener, A pGe semiconductor experiment showing chaos and hyperchaos, Physica D: Nonlinear Phenomena 35 (3) (1989) 425-435.

[20] M. Eiswirth, T.-M. Kruel, G. Ertl, F. Schneider, Hyperchaos in a chemical reaction, Chemical Physics Letters 193 (4) (1992) 305-310.

[21] M. P. Dafilis, F. Frascoli, P. J. Cadusch, D. T. J. Liley, Four dimensional chaos and intermittency in a mesoscopic model of the electroencephalogram, Chaos 23 (2) 023111.

[22] J. Rasmussen, E. Mosekilde, C. Reick, Bifurcations in two coupled Rössler systems, Mathematics and Computers in Simulation 40 (3-4) (1996) 247-270.

[23] L. Basnarkov, L. Kocarev, Forecast improvement in Lorenz 96 system, Nonlinear Processes in Geophysics 19 (5) (2012) 569575.

[24] T. Gao, Z. Chen, A new image encryption algorithm based on hyper-chaos, Physics Letters A 372 (4) (2008) 394-400.

[25] J.-G. Tong, Z.-X. Zhang, Q.-L. Sun, Z.-Q. Chen, Design of Wireless Sensor Network Node with Hyperchaos Encryption Based on FPGA, in: Chaos-Fractals Theories and Applications, 2009. IWCFTA '09. International Workshop on, 190-194, 2009.

[26] S. Nikolov, S. Clodong, Hyperchaos-chaos-hyperchaos transition in modified Rössler systems, Chaos, Solitons \& Fractals 28 (1) (2006) 252-263.

[27] Z. Qian, C. Zeng-Qiang, Y. Zhu-Zhi, Hyperchaos-Chaos-Hyperchaos Transition in a Class of On-Off Intermittent Systems Driven by a Family of Generalized Lorenz Systems, Chinese Physics Letters 25 (9) (2008) 3169.

[28] Z. Qian, C. Zeng-Qiang, Y. Zhu-Zhi, Blowout bifurcation and chaos-hyperchaos transition in five-dimensional continuous autonomous systems, Chaos, Solitons \& Fractals 40 (2) (2009) 1012-1020, ISSN 0960-0779.

[29] S. Dadras, H. R. Momeni, Four-scroll hyperchaos and four-scroll chaos evolved from a novel 4D nonlinear smooth autonomous system, Physics Letters A 374 (11-12) (2010) 1368-1373.

[30] Q. Li, S. Tang, X.-S. Yang, Hyperchaotic set in continuous chaos-hyperchaos transition, Communications in Nonlinear Science and Numerical Simulation 19 (10) (2014) 3718-3734.

[31] P. C. Rech, Chaos and hyperchaos in a Hopfield neural network, Neurocomputing 74 (17) (2011) 3361-3364.

[32] A. Wolf, J. B. Swift, H. L. Swinney, J. A. Vastano, Determining Lyapunov exponents from a time series, Phys. D 16 (3) (1985) 285-317.

[33] F. Waldner, R. Klages, Symmetric Jacobian for local Lyapunov exponents and Lyapunov stability analysis revisited, Chaos, Solitons \& Fractals 45 (3) (2012) 325-340.

[34] D. Wilczak, S. Serrano, R. Barrio, Coexistence and dynamical connections between hyperchaos and chaos in the 4D Rössler system: a Computer-Assisted proof, preprint . 
[35] T. Kapitaniak, Y. Maistrenko, S. Popovych, Chaos-hyperchaos transition, Physical Review E 62 (2) (2000) 1972.

[36] CAPD, Computer Assisted Proofs in Dynamics, a package for rigorous numerics, http://capd.ii.uj.edu.pl .

[37] H. Kokubu, D. Wilczak, P. Zgliczyński, Rigorous verification of cocoon bifurcations in the Michelson system, Nonlinearity 20 (9) (2007) 2147.

[38] D. Wilczak, P. Zgliczyński, Topological method for symmetric periodic orbits for maps with a reversing symmetry, Discrete and Continuous Dynamical Systems 17 (3) (2007) 629-652.

[39] D. Wilczak, Abundance of heteroclinic and homoclinic orbits for the hyperchaotic Rössler system, Discrete Contin. Dyn. Syst. Ser. B 11 (4) (2009) 1039-1055.

[40] D. Wilczak, Uniformly Hyperbolic Attractor of the Smale-Williams Type for a Poincaré Map in the Kuznetsov System, SIAM Journal on Applied Dynamical Systems 9 (4) (2010) 1263-1283.

[41] Z. Galias, W. Tucker, Rigorous study of short periodic orbits for the Lorenz system, in: Proc. IEEE Int. Symposium on Circuits and Systems, ISCAS'08, Seattle, 764-767, 2008.

[42] Z. Galias, Counting low-period cycles for flows, Int. J. Bifurcation and Chaos 16 (10) (2006) 2873-2886.

[43] P. Zgliczyński, Computer assisted proof of chaos in the Rössler equations and in the Hénon map, Nonlinearity 10 (1) (1997) $243-252$.

[44] P. Zgliczyński, M. Gidea, Covering relations for multidimensional dynamical systems, Journal of Differential Equations 202 (1) (2004) 32-58.

[45] A. Algaba, E. Freire, E. Gamero, A. Rodríguez-Luis, An exact homoclinic orbit and its connection with the Rössler system, Physics Letters A 379 (16-17) (2015) 1114-1121. 